\title{
A Model of Primitive Streak Initiation in the Chick Embryo
}

\author{
Karen M. Page*†, Philip K. Maini* $\ddagger$, Nicholas A. M. Monk§ and Claudio D. Stern $\|$
}

*Centre for Mathematical Biology, Mathematical Institute, 24-29 St Giles', Oxford OX1 3LB, U.K., $\dagger$ Institute for Advanced Study, Olden Lane, Princeton, NJ 08540, U.S.A., §Mathematical Modelling and Genetic Epidemiology Group, Division of Genomic Medicine, University of Sheffield, Royal Hallamshire Hospital, Sheffield S10 2JF, U.K. and \|Department of Genetics and Development, Columbia University, 701 West, 168th Street, New York, NY 10032, U.S.A.

(Received on 29 June 2000, Accepted in revised form on 2 November 2000)

\begin{abstract}
Initiation of the primitive streak in avian embryos provides a well-studied example of a pattern-forming event that displays a striking capacity for regulation. The mechanisms underlying the regulative properties are, however, poorly understood and are not easily accounted for by traditional models of pattern formation, such as reaction-diffusion models. In this paper, we propose a new activator-inhibitor model for streak initiation. We show that the model is consistent with experimental observations, both in its pattern-forming properties and in its ability to form these patterns on the correct time-scales for biologically realistic parameter values. A key component of the model is a travelling wave of inhibition. We present a mathematical analysis of the speed of such waves in both diffusive and juxtacrine relay systems. We use the streak initiation model to make testable predictions. By varying parameters of the model, two very different types of patterning can be obtained, suggesting that our model may be applicable to other processes in addition to streak initiation.
\end{abstract}

C) 2001 Academic Press

\section{Introduction}

The primitive streak is the first axial structure to appear during embryonic development in amniotes. It forms the scaffold on which the main body axis will be built. Its initiation is thus of fundamental importance in amniote embryonic development. In the chick embryo, the process of primitive streak initiation shows a remarkable capacity to regulate after experimental manipulation. This illustrates the fact that embryonic development is not simply an automated process pre-determined by the genetic code, but is highly adaptable. There are a number of models recently proposed to describe the spatiotemporal dynamics of streak formation (see e.g. Painter et al.,

† Author to whom correspondence should be addressed.
2000; Schnell et al., 2000). However, to our knowledge, there are no mathematical models proposed, as yet, to describe streak initiation. This is the aim of the present paper. We begin with a description of the relevant background biology.

\subsection{BACKGROUND BIOLOGY}

The embryo just prior to streak formation [stage XIII on the staging system of Eyal-Giladi \& Kochav (EG\&K), 1976] is a bilaminar disc (the blastodisc), composed of a single-cell thick epiblast underlain by a looser layer, the hypoblast. The diameter of the disc is approximately $2 \mathrm{~mm}$. The epiblast consists of a central region, the area pellucida or central disc, surrounded by a peripheral region underlain by large yolky cells, 
(a)

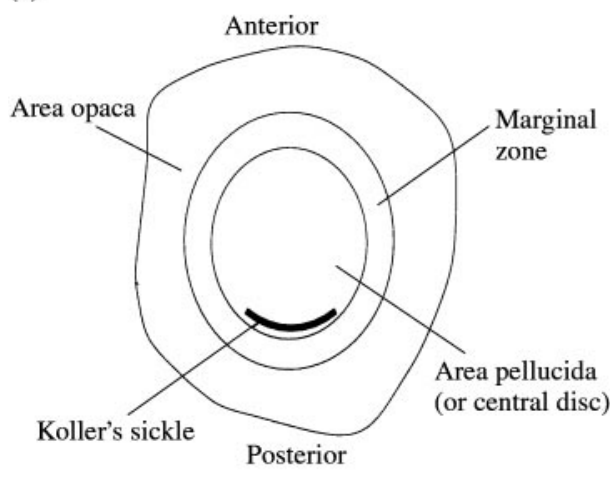

(b)

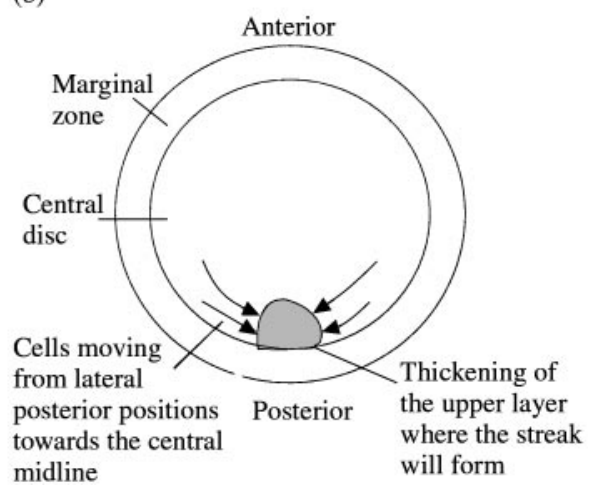

FIG. 1. (a) Bird's eye view of the blastodisc, showing the central disc (area pellucida), outer ring (area opaca) and marginal zone, which are present when the egg is laid at stage X. The position of Koller's sickle is also shown. (b) Cells move from posterior lateral parts of the area pellucida towards the central midline between stages X and XIV, and a thickening arises at the posterior edge of the epiblast at stage XIV-2 (the future primitive streak).

called the area opaca. Between the two regions is a ring of cells called the marginal zone, see Fig. 1(a).

At stage XIV (EG\&K), cells migrate from lateral posterior parts of the area pellucida towards the central midline [see Fig. 1(b)], accompanied by a thickening of cells in the posterior of the upper layer. This thickening forms the beginnings of the primitive streak. The streak then elongates anteriorly (becoming thinner) towards the centre of the blastodisc. It extends $60-75 \%$ of the way across the area pellucida. A groove forms in the middle of the streak and cells migrate from the epiblast at the sides of the streak, through the groove and into the cavity below. These cells form the mesodermal and endodermal layers of the embryo. A thickening, called Hensen's node, forms at the anterior end of the streak and cells also migrate through this node to become head process and notochordal mesoderm. The node then starts to regress posteriorly, leaving notochord structures in its wake.

\subsection{THE REGULATION OF THE CHICK BLASTODISC}

Although, under normal conditions, antero-posterior polarity is specified fairly early on by the action of gravity (see Kochav \& EyalGiladi, 1971), the axis is not irreversibly determined (i.e. external cues may alter its orientation) until the appearance of the streak itself. In fact, many manipulation experiments have been performed to alter the position of this axis or indeed to induce multiple axes.

Under normal circumstances the blastodisc develops a single primitive streak, which marks the position of the future backbone of the embryo. When the blastodisc is bisected, however, each half (provided it contains at least a portion of the marginal zone) develops its own streak (see Spratt \& Haas, 1960; Bachvarova et al., 1998). Thus, a simple cut is sufficient to trigger the development of twin embryos. Up to four streaks can be produced when the blastoderm is cut into quarters, one in each segment. The streak normally forms adjacent to the posterior marginal zone and there is evidence (see Bachvarova et al., 1998) that this structure causes streak induction. All (or at least most) of the marginal zone has the ability to initiate streak formation, but most of the circumference appears to do so only in the absence of a more potent inducer. (Indeed, if a second posterior marginal zone is transplanted into the lateral margin of a host embryo, then often only one of the two posterior marginal zones present will initiate axis formation; EyalGiladi, 1991.) Once the streak has started to form in the posterior, however, the rest of the marginal zone seems to lose permanently its initiation potential and/or the host its capacity to respond, such that, even if the posterior marginal zone and streak are removed, no new streak will be initiated (Khaner \& Eyal-Giladi, 1989; Eyal-Giladi \& Khaner, 1989; Bachvarova et al., 1998). This 
loss of potency/competence occurs almost simultaneously with the emergence of the streak in the posterior. This may be a coincidence and both may be triggered by earlier events, but assuming that the loss of potency is caused by a signal emanating from the posterior at the time of streak emergence, the signal must spread very rapidly throughout the marginal zone.

The cues which lead to the initiation of the primitive streak and to the regulative behaviour of the blastodisc are poorly understood. The identification of candidate primitive streak inducers is an active area of research (Shah et al., 1997; Cooke et al., 1994; Ziv et al., 1990).

\subsection{MODELLING PRIMITIVE STREAK INITIATION}

The capacity for small portions of the chick blastodisc to initiate the same number of streaks (one) as the whole blastodisc is difficult to explain using traditional models of embryonic pattern formation, such as reaction diffusion and mechanochemical models. These models do not naturally exhibit size regulation, the property of creating patterns which adapt to the size of the domain. In general, in these models, the number of pattern elements is proportional to the size of the domain. More complicated reaction-diffusion models can exhibit size regulation. For example, Meinhardt (1993) considered a reaction-diffusion model with a gradient source term and showed that it exhibited size regulation, with application to head formation in hydra. Othmer \& Pate (1980) showed how a reaction-diffusion model in which the diffusion coefficients were functions of the concentration of a control chemical could exhibit infinite scale invariance. However, none of these authors examined the time-scale on which patterns could plausibly form. Our studies, Page (1999), show that models based on straightforward reaction diffusion are likely to be too slow to be able to explain the regulation of the streak initiation process on a realistic time-scale.

Another feature of streak formation which is difficult to explain is the speed of the patterning process. When a blastodisc is cut into posterior and anterior halves, a localized patch of gene expression is initiated in the anterior portion of the marginal zone, marking the site of the future primitive streak, within approximately $3 \mathrm{hr}$, Skromne \& Stern (1998). Given that the circumference of the marginal zone is about $6 \mathrm{~mm}$ and that the diffusion coefficients of likely morphogens are very small in vivo, it is unlikely that this could be explained by a diffusive or reaction-diffusion mechanism (Page, 1999).

In this paper, we present a novel model of primitive streak initiation, which we believe can explain naturally the key experimental observations and could have much wider applicability in embryonic development. The model is described in Section 2 and the results of a travelling wave analysis (detailed in Appendix A) are summarized in Section 3 showing that, for realistic parameter values, this model is capable of generating propagating signals on the time-scale necessary to explain the available experimental results. In Section 4, we show that the model is consistent with the results of a number of experiments and in Section 5 we use the model to make experimentally testable predictions. We then discuss, in Section 6, the wider applicability of this type of model to pattern formation.

\section{The Model}

We propose a model mechanism in which marginal zone cells produce a streak-inducing chemical at a rate which is graded - higher posteriorly and lower anteriorly.* When the activator concentration reaches a threshold at a particular point in the domain, it causes the cells there to commit to differentiation, i.e. they are now destined to form part of the primitive streak. (It should be noted that the cells of the posterior marginal zone do not themselves become part of the streak, but rather cells just inside the marginal zone.) This commitment is irreversible. The committed cells produce an inhibitory factor at a constant rate. This inhibitor spreads as a wave throughout the domain preventing commitment of other cells in the epiblast. The inhibitor prevents commitment either directly (when it exceeds a certain threshold, the cells do not commit

\footnotetext{
* The posterior end appears to contain a higher density of cells (Kochav \& Eyal-Giladi, 1971), and hence we propose that production of the activator by these cells could lead to an appropriately graded production rate.
} 
even in the presence of super-threshold activator) or by degrading the activator.

Crucial to this model is a mechanism for the rapid passage of the inhibitor throughout the domain. There is evidence that cells can communicate via the release of diffusible substances (see Gurdon et al., 1994; Shimizu \& Gurdon, 1999; Slack, 1994) and that they can also signal to their immediate neighbours by presenting proteins on their surfaces which can bind to receptors on the neighbours' surfaces (see e.g. Collier et al., 1996; Zimmerman et al., 1993). The production of spatial gradients and of travelling waves by the latter mechanism is investigated in Monk (1998). In the context of the cell-inhibitory model of this paper, we investigate travelling waves occurring in both diffusive and juxtacrine signalling systems and assess the ability of each to explain the regulatory properties of the chick blastoderm.

\subsection{MODEL ASSUMPTIONS}

In addition to the general description of the model outlined above, we assume the following:

- The inhibitor either (i) diffuses and activates its own production locally, or (ii) activates its own production in neighbouring cells by juxtacrine signalling. There may be a delay between signalling and production of the inhibitor.

- The production rate of the inhibitor by the uncommitted cells is zero when the inhibitor concentration is zero and increases with that concentration, but always remains below a certain maximal rate.

- The activator and inhibitor both decay naturally.

- The inhibitor dynamics are fast relative to the activator dynamics, such that the wave of inhibition is rapidly triggered and spreads before much of the rest of the domain is activated.

\subsection{MODEL EQUATIONS}

We assume that the marginal zone is a thin ring which can be modelled as a one-dimensional domain with periodic boundary conditions. We denote by $u(x, t)$ and $v(x, t)$ the activator and inhibitor concentrations, respectively, at time $t$ and position $x$, measured from the posterior marginal zone (i.e. $x=0$ at the posterior).

We consider first the model mechanism in which the inhibitor degrades the activator and propagates via diffusion. The model equations are as follows:

$$
\begin{gathered}
u_{t}=\rho(x)-\gamma u-v u v+D_{u} u_{x x}, \\
v_{t}=k a+g(v)-\lambda v+D_{v} v_{x x}, \\
a(x, t)= \begin{cases}1 & \text { if } \max _{\tau<t}[u(x, \tau)] \geqslant u_{*}, \\
0 & \text { otherwise, }\end{cases}
\end{gathered}
$$

where $\rho(x)$ is the rate of production of activator at the point $x$ in the domain, $\gamma$ is the decay rate of the activator, $v$ is the reaction rate of the decay of the activator by the inhibitor, $D_{u}$ is the activator diffusion coefficient, $k$ is the rate at which the tissue made up of committed cells produces an inhibitor, $g(v)$ is a term describing the self-activation of the inhibitor, $\lambda$ is the linear decay rate of the inhibitor, $D_{v}$ is the inhibitor diffusion coefficient and $u_{*}$ is the activator threshold required in order to trigger cell commitment. The production term $\rho(x)$ is graded and is largest in the posterior. The variable $a(x, t)$ takes the value 1 if the cells at $x$ have committed to differentiation at time $t$ and otherwise takes the value 0 . This means that the first cells produce inhibitor only after they have experienced a super-threshold level of activator.

If instead the inhibitor blocks cell commitment directly then we drop the third term from the first equation $(v=0)$ and the last equation becomes

$a(x, t)= \begin{cases}1 & \text { if } \max _{\tau \in T_{v}(x, t)}[u(x, \tau)] \geqslant u_{*}, \\ 0 & \text { otherwise, }\end{cases}$

where $T_{v}(x, t)=\left\{\tau<t\right.$, s.t. $\left.v(x, \tau)<v_{* *}\right\}$, is the time period before $t$ and before the point $x$ has been inhibited from committing to differentiation and $v_{* *}$ is the threshold level of inhibitor at which cell commitment is blocked.

If the inhibition propagates via juxtacrine signalling and the inhibitor is confined to reside in the marginal zone then the second equation 
becomes

$$
\left(v_{i}\right)_{t}=k a_{i}+g\left(\bar{v}_{i}(t-\sigma)\right)-\lambda v_{i},
$$

where $v_{i}(t)$ represents the concentration of inhibitor at cell $i$ at time $t, a_{i}(t)$ is one if cell $i$ has committed to form streak at time $t$ and zero otherwise, $g$ is again a self-activation term, $\sigma$ is the delay between signalling and production of more inhibitor and $\bar{v}_{i}(t)$ represents the average inhibitor concentration in the cells neighbouring cell $i$. In the one-dimensional case considered here, $\bar{v}_{i}=\frac{1}{2}\left(v_{i-1}+v_{i+1}\right)$. Note that the model is now spatially discrete.

In setting up these model equations, we have assumed that the activator and committed cells reside in (or in the case of the cells, just inside-experiments show that posterior marginal zone cells do not actually contribute to the streak, Bachvarova et al., 1998) the marginal zone of the chick blastodisc. $\dagger$ This is approximately a circular domain and so for the purposes of simulation we consider the first and third equations of system (1) on a one-dimensional domain with periodic boundary conditions. Experimental evidence suggests (Spratt \& Haas, 1960) that the inhibitor probably resides in the central disc as well as the marginal zone. We initially analyse the case when the second equation is on a onedimensional domain with periodic boundary conditions, but then consider the second equation on a circular disc-shaped domain with zero flux boundary conditions (we suppose that the boundaries of this region are impermeable to the inhibitor). Initially, we have $u(x, t)=v(x, t)=$ $a(x, t)=0$.

We assume that $\rho(x)>\gamma u_{*}, \forall x$. (This means that each point in the marginal zone has the potential to initiate a streak). It is thus clear that the activator will reach threshold in the posterior and the inhibitor will then start to be produced. The pattern which results will depend crucially on whether a travelling wave of inhibition is established and, if so, on how fast the wave travels in comparison with the rate at which the activator reaches threshold at points in the domain distant from the posterior.

$\dagger$ There is strong evidence (Bachvarova et al., 1998; Spratt $\&$ Haas, 1960) that the streak-inducing signal comes from the marginal zone, see Section 1.2.
We thus analyse the inhibitor equation in isolation and treat the term $k a$ as a general localized source term. (If the inhibitory wave is established and is sufficiently fast then $a$ should remain zero except in the posterior.)

\section{Travelling Wave of Inhibition}

To study the travelling waves in isolation, we consider the equations

$$
v_{t}=s(x)+g(v)-\lambda v+D_{v} v_{x x}
$$

and

$$
\left(v_{i}\right)_{t}=s_{i}+g\left(\bar{v}_{i}(t-\sigma)\right)-\lambda v_{i}
$$

for the diffusive and the juxtracrine cases, respectively, where $s(x)$ and $s_{i}$ are the corresponding general localized source terms.

The form of the travelling wave is governed by (i) the form of $g$, (ii) the nature of the coupling, (iii) the domain geometry, and (iv) the parameter values. We summarize our results below. Full details are given in Appendix A.

(i) The form of $g$ : we have analysed the equation for two biologically plausible forms of $g$ - Heaviside and Hill function form. Both support travelling waves.

(ii) The form of the coupling: both types of coupling can give rise to fast propagating waves.

(iii) Domain geometry: in the case of diffusive coupling the speed of propagation on a twodimensional domain is slightly slower than that in one dimension. For the juxtracrine case the speed can be influenced by the geometry of the cell packing.

(iv) Parameter values: all cases considered exhibit sufficiently rapid travelling waves for biologically realistic parameter values.

\section{Numerical Simulations of the (full) Primitive Streak Model}

Once the inhibitory wave has been initiated in the posterior of the domain, the fate of the rest of the domain depends on a "race" at each point between reaching a superthreshold level of activator and being inhibited by the approaching wave. Consideration of the activator equation 
indicates that, for realistic parameters, activator diffusion can be neglected and hence that activator concentration ahead of the wave is approximately the same as if the inhibitor were everywhere zero. In the wake of the wave, even in the case of inhibitor degradation of the activator, cells which have not become committed by the time the wave reaches them have no possibility to do so. Thus, the commitment of a cell at a point $x$ in the domain really depends on whether the activator first reaches threshold there or whether the inhibitor wave reaches it. Straightforward analysis shows that if the inhibitory wave is established sufficiently quickly and moves fast enough, then the committed cells will be confined to a small region in the posterior.

We simulate the full model equations [system (1)] to see if realistic parameters give rise to an inhibitory wave which is sufficiently fast to confine commitment to a small region in the posterior, as is suggested by experimental results. We also test that the model correctly predicts the outcome of the following manipulation experiments:

1. Bisecting the blastodisc perpendicular to the anterior-posterior axis: experiments show that this normally results in a streak forming from the posterior end of the posterior half (its normal position in the whole embryo), but also a streak forming from one of the lateral margins of the anterior half. In some cases, the anterior half may form a streak from its anterior margin or two streaks, one from each lateral margin (Spratt \& Haas, 1960).

2. Implanting glass beads to replace the posterior marginal zone: this generally results in streaks forming on either side of the beads, which are implanted simply to prevent healing of posterior marginal zone (Eyal-Giladi, 1991).

3. Implanting a second posterior marginal zone from a donor embryo into the lateral margin of the host blastodisc: this often results in only one of the posterior marginal zones initiating a streak. This competition between posterior marginal zones only occurs if the implant is sufficiently close to the original marginal zone. Which of the posterior marginal zones is successful depends on the size of the implant (the bigger it is the more likely it is to succeed) and the relative ages of the implants (younger posterior marginal zones seem to be more successful; Eyal-Giladi, 1991).

We assume that when these experiments were performed on real blastodiscs, the manipulation occurred (stages X-XIII on the staging system of Eyal-Giladi and Kochav) before commitment had taken place in the posterior.

We assume, for ease of simulation, that the inhibitor resides in the marginal zone rather than the central disc. This means that the waves of inhibition take slightly longer to reach their targets and so slightly more of the cells will commit to differentiation. On balance, experiments suggest that the inhibitor actually spreads throughout the central disc, but this makes only a small difference to the speed of propagation (as our calculations in Appendix A show) and will only dramatically affect the result of the glass bead implant experiment.

We compare direct inhibition of cell commitment with degradation of the activator and propagation of the inhibitory wave via a diffusive mechanism with propagation via juxtacrine signalling mechanisms.

First, we consider the system in which the inhibitor diffuses and directly blocks cell commitment. The domain length (circumference of the blastodisc) is about $6 \mathrm{~mm}$ and we take the inhibitor diffusion coefficient to be as large as is realistic, approximately $1.0 \times 10^{-7} \mathrm{~cm}^{2} \mathrm{~s}^{-1}$. If we nondimensionalize such that the domain becomes $[0,1]$ (the domain is periodic and the points 0 and 1 correspond to the posterior of the marginal zone, whilst the point $\frac{1}{2}$ corresponds to the anterior) and with a time-scale of $1000 \mathrm{~s}$, then the non-dimensional value of $D_{v}$ is about $3.0 \times 10^{-4}$. For purposes of illustration, we take the activator diffusion coefficient to be 10 times smaller (this is simply because we have taken the inhibitor diffusion coefficient to be as large as possible). We take the threshold level of activator, $u_{*}$, and the threshold for self-activation of the inhibitor $v_{*}$ both to be 1.0 in our arbitrary concentration units. We take the threshold for inhibition of cell commitment, $v_{* *}$, to be 0.5 . In order for a travelling wave to be possible, we require $\mu / \lambda>v_{*}=1.0$, and we also want the inhibitor kinetics to be much faster than the activator kinetics. We choose $\mu=500.0, \lambda=10.0$ and 

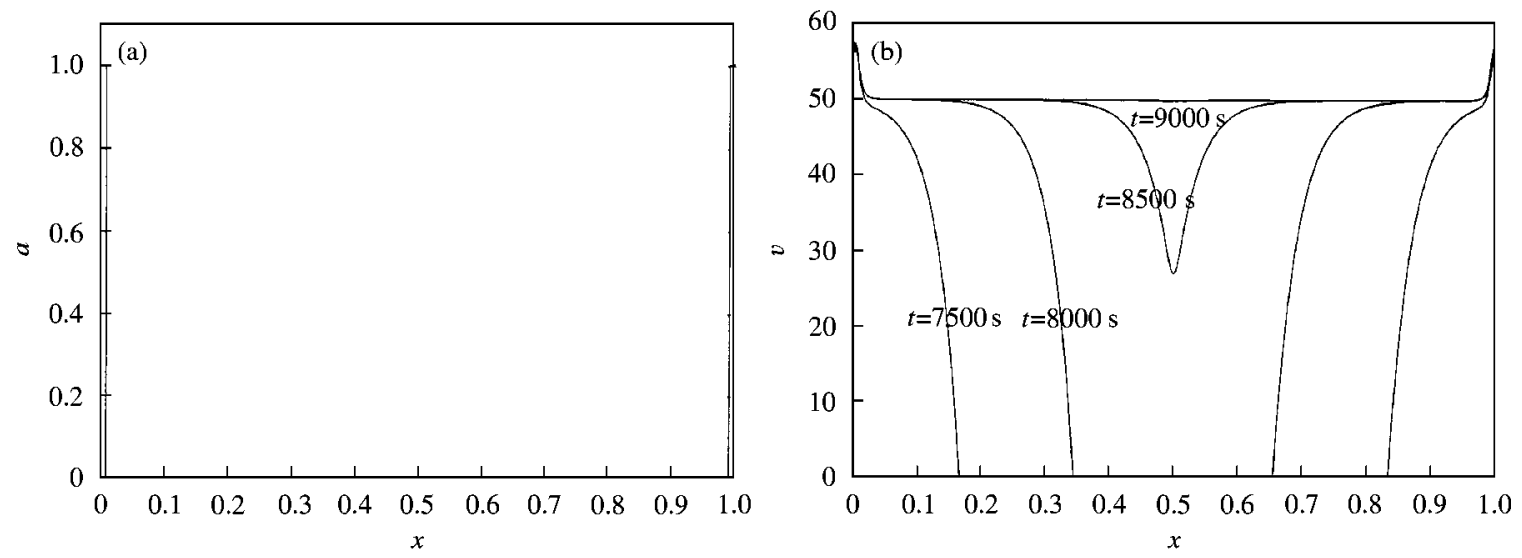

FIG. 2. (a) Final steady-state committed cell profile, $a(x)$, from a numerical simulation of the full model (1), with diffusive inhibitor which directly blocks cell commitment. Parameter values as given in the text. A discretization of 2001 points was used. (b) Inhibitor profile at times $t=7500,8000,8500$ and $9000 \mathrm{~s}$ in the simulation described in (a).
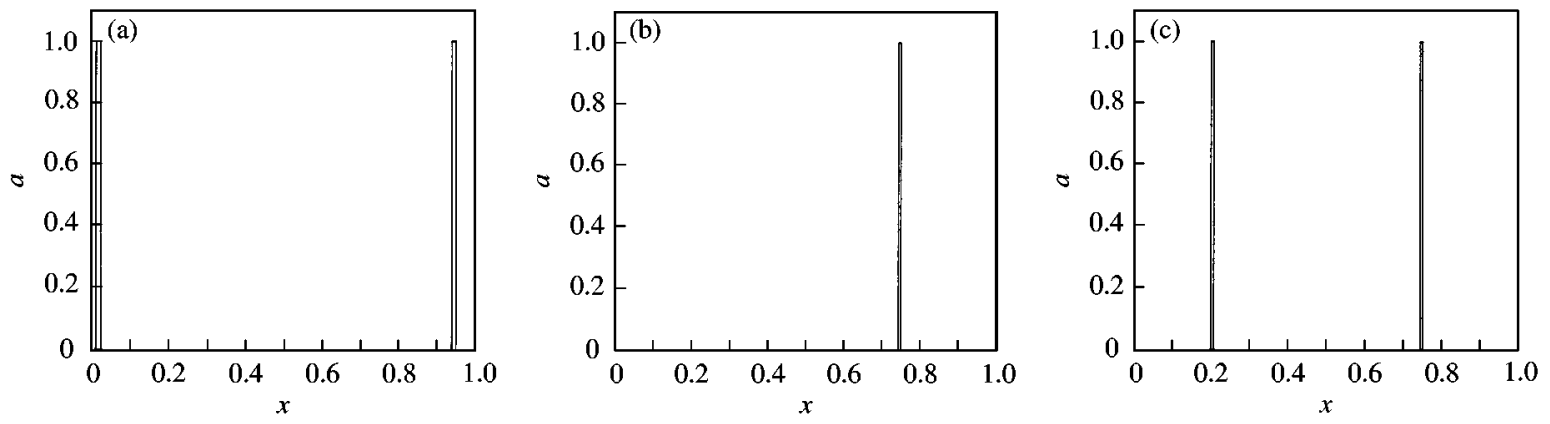

FIG. 3. Final committed cell profile in the anterior portion of the blastodisc in which the blastodisc was cut after a time $t=5000 \mathrm{~s}$. (a) The remaining portion is [0.01, 0.95], (b) the remaining portion is $[0.35,0.75]$, (c) the remaining portion is $[0.2,0.75]$. Parameter values $D=0.0001, \mu=10.0, \lambda=1.0$ and $v_{*}=1.0$. See text for details.

$k=100.0$. In dimensional terms, the inhibitor has a half-life of $100 \mathrm{~s}$. The activator dynamics should be much slower and in order for each point in the domain to have the potential to initiate streak, we require $\rho(x)>\gamma u_{*}, \forall x$. We choose $\gamma=0.1$ (corresponding to a half-life of around $3 \mathrm{hr}$ ) and

$$
\rho(x)= \begin{cases}0.2(1-x), & x<\frac{1}{2}, \\ 0.2 x & x \geqslant \frac{1}{2} .\end{cases}
$$

Figure 2(a) shows the value of $a$ plotted against $x$ at time $t=8000 \mathrm{~s}(x=0$ and 1 correspond to the posterior). The form of $a$ (corresponding to a small patch of determined cells in the posterior) remains constant thereafter. Determination occurs at $t \approx 7000 \mathrm{~s} \approx 2 \mathrm{hr}$. Figure 2 (b) shows the inhibitor concentration profile at various times after determination occurs in the posterior.

Figures 3(a)-(c) show the final steady-state profiles of $a$ when the blastodisc was cut at time $t=5000 \mathrm{~s}$ in various different places. Figure 3(a) corresponds to the glass beads experiment in which the posterior marginal zone is excised (and replaced with beads to prevent healing). The portions of the domain $[0,0.01]$ and $[0.95,1.0]$ were excised, so that the excised portion was not symmetrical about the anterior-posterior axis. Zeroflux boundary conditions were imposed at the cut edges. Nevertheless, a streak formed on either side of the cut. Differentiation had occurred by $t=8000 \mathrm{~s}$. Figures 3(b) and (c) show the final profile of $a$ in the anterior half of the blastodisc when the posterior half is excised. In Fig. 3(b), the anterior half consists of the portion of the domain 

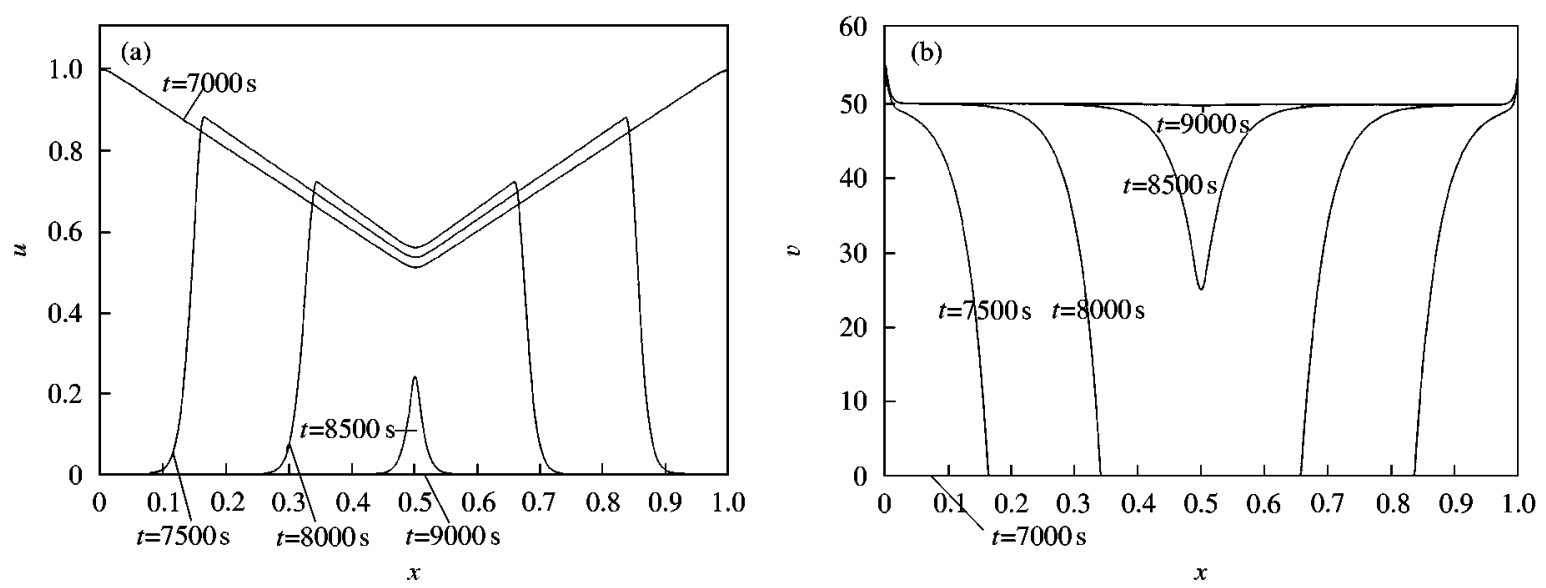

FIG. 4. (a) Activator profile and (b) inhibitor profile at times $t=7000,7500, \ldots, 9000 \mathrm{~s}$ in a simulation of the system with a diffusive inhibitor which degrades the activator $(v=1.0)$. Other parameters as in Fig. 3. See text for details.

$[0.35,0.75]$ and only one streak forms at $x=0.75$, which comes from the posterior-most part of the blastodisc. In Fig. 3(c), the anterior half is more symmetrical and larger, consisting of [0.2, 0.75], and two streaks form. The time taken before cells committed to form a streak in the anterior half was about 6000 or $7000 \mathrm{~s}$ after the cut was made. This is less than $2 \mathrm{hr}$. However, the time at which commitment to form a streak in the anterior half takes place is later than it would do so in the posterior in an unmanipulated blastodisc. This agrees with the results of experimental manipulations.

We repeat the simulations of Figs 2 and 3, with the same parameters, but with inhibitor degradation of the activator $(v=1.0)$ rather than direct inhibition of cellular commitment. The sites of streak initiation are the same in all cases, although these sites are slightly narrower. However, we can alter the width of these sites by altering $v_{* *}$ or $v$. Small $v_{* *}$ and large $v$ lead to narrow sites of committed cells. In Fig. 4, we show the activator and inhibitor concentration profiles at different times. This shows that the activator concentration ahead of the wave of inhibition is largely unaffected by the inhibitor (see Section 3) and that behind the wave it decays rapidly.

We now look at the system with juxtacrine signalling and no inhibitor diffusion. The activator diffusion seems irrelevant to the mechanism of our model and so, for ease of simulation, we assume $D_{u}=0$. We take the other parameters to have the same values as in Fig. 2. We take a self-activation term $\mu \mathscr{H}\left(\bar{v}_{i}-v_{*}\right)$, where $\bar{v}_{i}=\frac{1}{2}\left(v_{i-1}+v_{i+1}\right)$. We divide the domain [0, $6 \mathrm{~mm}$ ] into 2000 cells. This assumes that the cells have diameter approximately $3 \mu \mathrm{m}$. Again, we obtain cell commitment in exactly the same places as in Figs 2 and 3. The committed regions are a few cells thick.

Thus, the model either with diffusive propagation of the inhibitor or with juxtacrine signalling and either with inhibitor degradation of the activator or with direct blocking of cell commitment is capable of explaining normal initiation of one primitive streak in the whole blastoderm and the regulation that occurs when the blastodisc is bisected or has its posterior marginal zone excised (for a similar example of a model for generating size-regulating patterns, see Green $\&$ Cooke, 1991). It can also explain the relatively short time-scales in which these events occur.

The model also predicts that the anterior marginal zone will permanently lose its ability to induce a streak once the wave of inhibition from the posterior has entered the anterior half. This clearly occurs after commitment in the posterior. Experiments suggest that the marginal zone loses its potential to initiate a streak, even in the absence of the posterior marginal zone, once the streak has started to form in the posterior, see Khaner \& Eyal-Giladi (1989), Eyal-Giladi \& Khaner (1989) and Bachvarova et al. (1998). The initial appearance of the streak and the loss of potency of the marginal zone seem to be almost 
exactly coincident to within experimentally determinable limits.

If a second posterior marginal zone is implanted into the lateral margin of a host embryo, provided the rate at which cells in each posterior marginal zone produce activator is somewhat different (for example, if activator production rate is age-dependent and the embryos are of slightly different ages) and the separation of the two posterior marginal zones is not too great then, according to the model, competition will occur and only one streak will be induced. If the posterior marginal zones are too similar in their production rate and level of activator concentration or if they are too far apart then two streaks will form, as observed experimentally.

\section{Model Predictions}

The model presented here predicts that, in an embryo which is defective for the inhibitor, the whole of the marginal zone will induce streak formation. The streaks in the mutant should form in order, from posterior to anterior.

The axin mutant mouse has a phenotype which induces multiple streaks emanating from all regions of the margin, see Zeng et al. (1997). (Axin blocks the Wnt signalling pathway, which has been proposed to have a role in streak induction in the chick, see Cooke et al., 1994.) Since axin is an intracellular molecule, we propose that the inhibitor may be a secreted antagonist of Wnt or nodal signalling, such as frzb, Dkkl, cerberus or crescent (Baranski et al., 2000; Zhu et al., 1999; Pfeffer et al., 1999).

The behaviour of our model is characterized by processes operating on two distinct time-scales. Initially, there is a slow increase of activation across the whole region with a peak at the posterior, then, once the most posterior cells have committed to form primitive streak, there is initiation of a wave of inhibition that rapidly sweeps across the whole domain. During the first phase, there exists a posterior to anterior gradient of streak-initiating potential consistent with experimental observations suggesting that lateral segments are better inducers than anterior segments. During the second phase, there is a loss of streak-forming competence which results from the wave of inhibition. Hence, the model predicts that during this short-time window anterior segments lose their streak-forming competence later than lateral segments.

We propose an experiment in which a stage XIV anterior half, whose marginal zone has lost the capacity to initiate a streak, is grafted on to a stage $\mathrm{X}$ anterior half. According to the model, the cells in the older anterior half should have been inhibited and should cause the production of the inhibitor in the younger anterior half, thus preventing a streak from being initiated there.

Finally, we propose an experiment which should be able to distinguish between a Turing mechanism of streak initiation (in which the activator and inhibitor react and diffuse and become patterned via a diffusive instability) and the cell-inhibitory model with diffusive inhibitor, provided that diffusion in both model mechanisms occurs through gap junctions. This is very plausible, since the epiblast of the blastodisc is a coupled epithelium (Stern \& Mackenzie, 1983). (In the case of the Turing mechanism, it is extremely likely that the activator would diffuse through gap junctions, since it must somehow be confined to the marginal zone.) The experiment involves the drug-induced blocking of gap junctional communication, see Levin \& Mercola (1998, 1999). The crucial difference between the models is that the activator diffusion is an important component in the Turing mechanism, but not in the cell-inhibitory mechanism. In the Turing scenario, we predict that the activator and inhibitor would remain at their uniform steady-state values and not induce a primitive streak, whereas in the cell-inhibitory model the activator would reach threshold at each point before any inhibitor was produced there. Hence, streaks would form from all sites round the marginal zone.

\section{Further Features of the Model}

We have shown that the model is capable of explaining many of the experimental results relating to streak initiation. We find also that it is capable of exhibiting very different behaviour and producing periodic patterns. We simulate the model equations with a diffusive inhibitor and inhibitor degradation of the activator. We use the same parameter values as before, except that we 

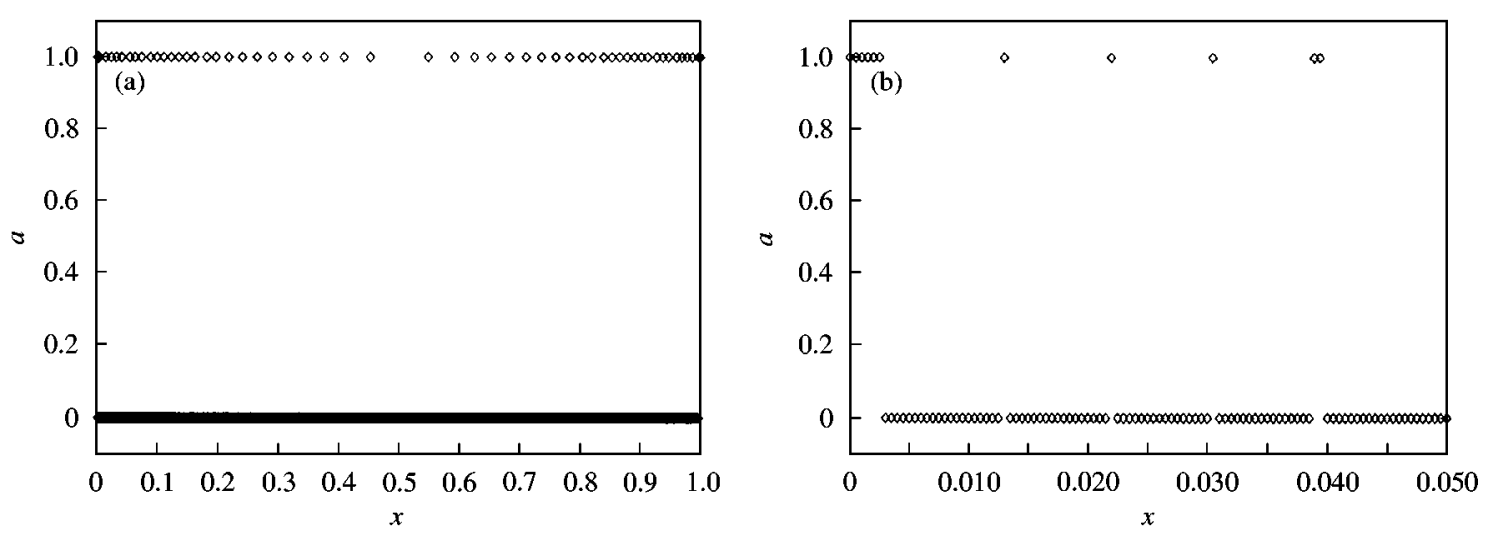

FIG. 5. (a) Final committed cell profile in a numerical simulation of the full model with a diffusive inhibitor which degrades the activator. Parameters given in the text. A discretization of 2001 points (each point corresponds to a cell) was used. (b) A blown-up version of the profile on $[0,0.05]$.

set $\mu=10.0$ instead of 500.0. This means that a forward-propagating travelling wave of inhibition cannot be set up. We use a discretization of 2001 points. Thus, each point corresponds roughly to a cell in the biological system. In Fig. 5(a), we show the final steady-state profile of $a$. We consider each point in the domain to correspond to a cell and so those points at which $a=1$ correspond to cells committed to differentiation and those at which $a=0$ correspond to uncommitted cells. Figure 5(b) shows the profile on the section of the domain $[0,0.05]$. This shows that approximately one cell in every 20 becomes committed to differentiation. Although similar patterns can be produced by a typical activator-inhibitor Turing system, the novelty here is that these patterns have been produced by a size-regulating model on changing one parameter.

We repeat the simulation of Fig. 5 with direct blocking of cellular commitment $(v=0)$. When $v_{* *}=0.5$, approximately one cell in every four commits to differentiation. With $v_{* *}=0.05$, a pattern similar to that in Fig. 5 is produced, with roughly every 20 cell committed to differentiation. The spacing between committed cells increases away from the posterior (region of greatest activator production).

A simulation with a juxtacrine coupling, no inhibitor diffusion and inhibitor degradation of the activator $(v=1.0)$ leads to a pattern in which there is a small patch of committed cells in the posterior, followed by a patch of alternating committed and uncommitted cells. Nearer the anterior, committed cells become sparser until in the anterior there are no committed cells. Such patterns in which the frequency of committed cells decreases towards the anterior appear to be typical in relay systems. The patterns which form are probably dependent on the juxtacrine coupling. Our system employs a very simple coupling. For more sophisticated couplings, see Monk (1998), Owen \& Sherratt (1998) and Owen et al. (1999). Further investigation of the types of patterns that could develop in similar systems in two dimensions, with various forms of cellular coupling, would be interesting. Even with our simple coupling, however, the system produced finegrained structures with period greater than two cells. This is a property that many juxtacrine models do not have (see Collier et al., 1996) and may have important biological applications.

\section{Discussion}

The initiation of the primitive streak in the chick embryo is a fundamental developmental event. The mechanisms involved in the initiation are, as yet, poorly understood. Experimental manipulations of the chick blastodisc reveal that the initiation process displays size regulation. In most cases, an isolated portion of the blastodisc, provided it contains part of the marginal zone, will initiate exactly one streak. Although this property of size regulation can be exhibited by modified reaction-diffusion models (Meinhardt, 
1993; Othmer \& Pate, 1980) it is not clear if this can occur on a sufficiently fast time-scale for primitive streak initiation. Models based on straightforward diffusion or on reaction-diffusion are likely to be too slow to be able to explain the regulation of the streak initiation process on a realistic time-scale (Page, 1999). In this paper, we sought an alternative model of primitive streak initiation which could display its key features more naturally. We consider that this model could have wider applicability in embryonic development.

The mechanism involves cells producing an inducing agent which, on reaching a threshold, causes cellular commitment to form part of the primitive streak. Committed cells produce an inhibitor which activates its own production in uncommitted cells and passes as a wave throughout the domain, preventing commitment at other sites. Such waves of inhibition are well documented in other areas of developmental biology, such as calcium waves in fertilized eggs - see, for example, Busa \& Nuccitelli (1985); Gilkey et al. (1978); Lane et al. (1987) and Jaffe (1999). The model is intrinsically different from a reaction-diffusion model in that cells have a memory - their commitment is irreversible. An important feature of the model is that whilst straightforward diffusion of an inhibitor would be too slow to be biologically realistic (see Crick, 1970), a diffusive (or indeed juxtacrine) travelling wave can pass much more rapidly throughout the domain. In a standard reaction-diffusion model a travelling wave could not pattern the blastodisc, since the final steady state left behind the wave would be uniform and hence cells would ultimately reach the same state of differentiation everywhere. The cell memory property is thus a crucial element of the model proposed here.

We began the analysis of the model by assessing the speed of propagation of (inhibitory) travelling waves in a system with self-activation and either diffusive or juxtacrine coupling. We found that if a travelling wave arose then it had a speed uniquely determined by the parameters of the system. This suggests that mechanisms of this type could allow cells to communicate over fixed distances on a predictable time-scale. For the case in which the self-activation of the inhibitor takes a caricature Heaviside function form, we were able to calculate the wave speed analytically for the cases of both diffusive and juxtacrine coupling. It is an open question as to how to determine wave speeds in juxtacrine signalling systems with general self-activation. This caricature model serves to give insight into the importance of different parameters in determining the wave speed and to estimate the order of magnitude of possible wave speeds. We found good agreement between the analytical results and those of numerical simulations.

Our simulations show that in both the diffusive and juxtacrine cases, more realistic forms of the self-activation term led to waves which were slightly slower than, but with speeds of the same order of magnitude as those of the caricature model. In two dimensions, the diffusive travelling wave was slower than its one-dimensional counterpart very near to a localized source, but beyond a short distance from the source the difference became negligible. In the juxtacrine system, the two-dimensional problem was more complex. The propagation of the wave was influenced by the positions of the cells and the numbers of orientations of the nearest-neighbour interactions. We found that with the simple coupling used in our model, the wave speed was considerably reduced compared to its one-dimensional counterpart, because cells had to share their signalling between more neighbours and hence forwards signalling was reduced. We postulate, however, that this might not be the case for other more complicated couplings. The propagation of waves in a juxtacrine relay system in two dimensions with various different couplings warrants further investigation.

Considering realistic parameters, we found that a diffusive inhibitory wave could probably have a speed of up to about $10^{-2} \mathrm{~mm} \mathrm{~s}^{-1}$ and hence pass from the posterior to the anterior of the embryo in less than $5 \mathrm{~min}$. In the case of the juxtacrine relay model, a wave of inhibition could sweep across the domain in a similar time, provided there exists a quick-release mechanism (see, e.g. Lyons et al., 1988) for the inhibitor.

Analysis of the activator equation and numerical simulations of the full model showed that if the speed of propagation of the inhibitory wave were fast enough, the cells would commit to differentiation only in a small region in the 
posterior. Numerical simulations of the model predict that it can explain the regulation that occurs in primitive streak initiation and that it can explain initiation on a realistic time-scale. This is independent of whether the inhibitor propagates via juxtacrine signalling or via diffusion and of whether the inhibitor blocks cell commitment directly or does so by degrading the activator.

The model gives rise to a number of testable predictions, as detailed in the previous section. The streak-inducing agent itself has not yet been positively identified, although a number of candidates have been found - perhaps the most promising being the $\mathrm{Vg} 1$ protein, acting together with Wnt.

The model has the ability to give rise to a single structure in domains of various sizes on time-scales which are appropriate for biological development. We propose that this feature makes this model relevant to many other biological systems.

Many other instances occur in development in which the emergence of a structure inhibits formation of further (identical) structures elsewhere in the field of interest. For example, the freshwater polyp Hydra normally has only one head and foot, but if bisected regenerates the appropriate body part at the cut end of each half (see Gierer \& Meinhardt, 1972; Meinhardt, 1993; Sherratt et al., 1995). Another fascinating example is the initiation of limbs in the tailbud stage salamander Ambystoma maculatum. In this case, each limb disc normally produces one limb, but if the limb disc is split into vertical segments by placing thin barriers which prevent reunion, then each segment produces a distinct limb. A similar phenomenon was observed in a pond in Santa Cruz, CA, in which many-legged frogs and salamanders were found. It was discovered that this was due to an infestation of parasitic trematode worms whose eggs split the limb buds of the tadpoles into several parts [see Gilbert (1991), which refers to the then unpublished data of Sessions (1998) and Sessions et al. (1999)].

The model system can also produce periodic short-wavelength spatial patterns if the parameters are such that a travelling wave of inhibition is not triggered. This gives rise to the intriguing possibility that by altering parameters of the system the same mechanism could have evolved to give rise to both size regulating and fixed wavelength periodic patterns. It would be very interesting to investigate more thoroughly the types of periodic patterns that can be produced by this system, especially in two dimensions, looking also at the effects of different couplings in the juxtacrine case. In the one-dimensional system, we found that the pattern spread out as a wave from the "posterior" (fastest activatorproducing) end to the "anterior" end. This sequential formation of pattern occurs in many biological patterning processes. Examples include the formation of stripes on the back of the Alligator mississippiensis, somites in amniotes and chick feather primordia.

In general, we have found that the novel model proposed in this paper can give rise to two highly different types of pattern on biologically realistic time-scales and could be applicable to a very wide range of developmental patterning events.

KMP would like to thank the Wellcome Trust for funding under the Prize Studentship Scheme in Mathematical Biology, Grant Number 047910. She would also like to acknowledge support from the Leon Levy and Shelby White Initiatives Fund, the Florence Gould Foundation, the J. Seward Johnson, Sr. Charitable Trusts, the Ambrose Monell Foundation and the Alfred P. Sloan Foundation.

NAMM gratefully acknowledges the support of the Royal Society and BBS CDS's, research on this topic was funded by the National Institute of Health (GM 56656)

\section{REFERENCES}

Bachvarova, R. F., Skromne, I. \& Stern, C. D. (1998). Induction of primitive streak and Hensen's node by the posterior marginal zone in the early chick embryo. Development 125, 3521-3534.

Baranski, M., Berdougo, E., SAndler, J. S., Darnell, D. K. \& BURRUS, L. W. (2000). The dynamic expression pattern of frzb-1 suggests multiple roles in chick development. Dev. Biol. 217, 25-41.

BusA, W. B. \& Nuccitelli, R. (1985). An elevated free cytosolic $\mathrm{Ca}^{2+}$ wave follows fertilization in the eggs of the frog Xenopus laevis. J. Cell. Biol. 100, 1325-1329.

Collier, J. R., Monk, N. A. M., Maini, P. K. \& Lewis, J. H. (1996). Pattern formation by lateral inhibition with feedback: a mathematical model of Delta-Notch intercellular signalling. J. theor. Biol. 183, 429-446.

Cooke, J., TAkada, S. \& McMahon, A. (1994). Experimental control of axial pattern in the chick blastoderm by local expression of Wnt and activin - the role of HNK-1 positive cells. Dev. Biol. 164, 513-527. 
CRICK, F. (1970). Diffusion in embryogenesis. Nature 225, 420-422.

Dyson, S. \& GuRdon, J. B. (1998). The interpretation of position in a morphogen gradient as revealed by occupancy of activin receptors. Cell 93, 557-568.

EYAL-GILADI, H. (1991). The avian marginal zone and its role in early development. In: Formation and Differentiation of Early Mesoderm, pp. 9-21. NATO ASI Series.

Eyal-Giladi, H. \& KHANER, O. (1989). The chick's marginal zone and primitive streak formation. 2. Quantification of the marginal zone's potencies - temporal and spatial aspects. Dev. Biol. 134, 215-221.

Eyal-Giladi, H. \& Kochav, S. (1976). From cleavage to primitive streak formation: a complementary normal table and a new look at the first stages of the development of the chick. Dev. Biol. 49, 321-337.

FerRell, J. E. \& MACHLEDER, E. M. (1998). The biochemical basis of an all-or-none cell fate switch in Xenopus oocytes. Science 280, 895-898.

Gierer, A. \& MEINHARDT, H. (1972). A theory of biological pattern formation. Kybernetik 12, 30-39.

Gilbert, S. F. (1991). Developmental Biology, Sinauer, 3rd Edn.

Gilkey, J. C., JAFFe, L. F., Ridgeway, E. G. \& Reynolds, G. T. (1978). A free calcium wave traverses the activating egg of the medaka, oryzia latipes. J. Cell Biol. 76, 448-466.

Green, J. A. B. \& CoOKE, J. (1991). Induction, gradient models and the role of negative feedback in body pattern formation in the amphibian embryo. Sem. Dev. Biol. 2, 95-106.

Grindrod, P. (1996). The Theory and Application of Reaction-Diffusion Equations. 2nd Edn. Oxford: Clarendon Press. Gurdon, J. B., Harger, P., Mitchell, A. \& Lemaire, P. (1994). Activin signalling and response to a morphogen gradient. Nature 371, 487-492.

HiLl, A. V. (1910). Possible effects of the aggregation of the molecules of haemoglobin on its dissociation curves. $J$. Physiol. 40, iv-viii.

JAFFE, L. F. (1999). Organization of early development by calcium patterns. Bioessays 21, 657-667.

KHANER, O. \& EyAl-GilADI, H. (1989). The chick's marginal zone and primitive streak formation. 1. coordinative effect of induction and inhibition. Dev. Biol. 134, 206-214.

Kochav, S. \& EYAL-GILADI, H. (1971). Bilateral symmetry in chick embryo determination by gravity. Science 171, 1027-1029.

Lane, D. C., Murray, J. D. \& Manoranjan, V. S. (1987). Analysis of wave phenomena in a morphogenetic mechanochemical model and an application to postfertilisation waves on eggs. IMA J. Math. Appl. Med. Biol. 4, 309-331.

Levin, M. \& Mercola, M. (1998). Gap junctions are involved in the early generation of left-right asymmetry. Dev. Biol. 203, 90-105.

Levin, M. \& Mercola, M. (1999). Gap junction-mediated transfer of left-right patterning signals in the early chick blastoderm is upstream of shh asymmetry in the node. Development 126, 4703-4714.

Lyons, R. M., Keski-OJA, J. \& Moses, H. L. (1988). Proteolytic activation of latent transforming growth factor- $\beta$ from fibroblast-conditioned medium. J. Cell Biol. 106, 1659-1665.

MEINHARDT, H. (1993). A model for pattern formation of hypostome, tentacles, and foot in hydra: how to form structures close to each other, how to form them at a distance. Dev. Biol. 157, 321-333.

Monk, N. A. M. (1998). Restricted-range gradients and travelling fronts in a model of juxtacrine cell relay. Bull. Math. Biol. 60, 901-918.

Murray, J. D. (1989). Mathematical Biology, Biomathematics Texts, Vol. 19. Berlin: Springer-Verlag.

OthmeR, H. G. \& PATE, E. (1980). Scale-invariance in reaction-diffusion models of spatial pattern formation. PNAS 77, 4180-4184.

Owen, M. R. \& Sherratt, J. A. (1998). Mathematical modelling of juxtacrine cell signalling. Math. Biosci. 152, $125-150$.

Owen, M. R., Sherratt, J. A. \& Myers, S. R. (1999). How far can a juxtacrine signal travel? Proc. Roy. Soc. Lond. B 226, 579-585.

PAGE, K. M. (1999). Mathematical models in embryology: the selection, regulation and speed of formation of patterns. DPhil.

Painter, K. J., Maini, P. K. \& Othmer, H. G. (2000). A chemotactic model for the advance and retreat of the primitive streak. Bull. Math. Biol. 62, 501-525.

PAPIN, C. \& SMith, J. C. (2000). Gradual refinement of activin-induced thresholds requires protein synthesis. Dev. Biol. 217, 166-172.

Pfeffer, P. L., DeRobertis, E. M. \& IzPisuaBelmonte, J. C. (1999). Crescent, a novel chick gene encoding a frizzled-like cysteine- rich domain, is expressed in anterior regions during early embryogenesis. Int. J. Dev. Biol. 41, 449-458.

Schnell, S., Painter, K. J., Maini, P. K. \& Othmer, H. G. (2000). Spatiotemporal pattern formation in early development: a review of primitive streak formation and somitogenesis. In: Mathematical Models for Biological Pattern Formation. (Maini, P. K., Othmer, H. G., ed.), IMA Volumes in Mathematics and its Application 121, 11-37.

SEGEL, L. A. (1991). Biological Kinetics, 1st Edn. Cambridge: Cambridge University Press.

Sessions, S. K. (1998). Frog deformities. Science 279, 461-462. Sessions, S. K., Franssen, R. A. \& Horner, V. L. (1999). Morphological clues from multilegged frogs: are retinoids to blame? Science 284, 800-802.

Shah, S. B., Skromne, I., Hume, C. R., Kessler, D. S., LeE, K. J., STERn, C. D. \& DodD, J. (1997). Misexpression of chick $\mathrm{Vg} 1$ in the marginal zone induces primitive streak formation. Development 124, 5127-5138.

Sherratt, J. A., Maini, P. K., Jaeger, W. \& Mueller, W. A. (1995). A receptor based model for pattern formation in hydra. Forma 10, 77-95.

ShimizU, K. \& GuRdon, J. B. (1999). A quantitative analysis of signal transduction from activin receptor to nucleus and its relevance to morphogen gradient interpretation. PNAS 96, 6791-6796.

Skromne, I. \& STERN, C. D. (1998). Unpublished results.

SLACK, J. M. W. (1994). How to make the gradient. Nature 371, 477-478.

SPRATT, N. T. \& HAAS, H. (1960). Integrative mechanisms in development of the early chick blastoderm. I. Regulative potentiality of separate parts. J. Exp. Zool. 145, 97-137.

Stern, C. D. \& MackenzIE, D. O. (1983). Sodium-transport and the control of epiblast polarity in the early chick-embryo. J. Embryol. Exp. Morph. 77, 73-98.

Zeng, L., FAgOtTO, F., Zhang, T., HsU, W., VAsiceK, T. J., Perry, W. L., LeE, J. J., Tilghman, S. M., 
Gumbiner, B. M. \& Costantini, F. (1997). The mouse fused locus encodes axin, an inhibitor of the Wnt signalling pathway that regulates embryonic axis formation. Cell 90, 181-192.

Zhu, L., Marvin, M. J., Gardiner, A., Lassar, A. B., Mercola, M., Stern, C. D. \& Levin, M. (1999). Cerberus regulates left-right asymmetry of the embryonic head and heart. Curr. Biol. 9, 931-938.

Zimmerman, G. A., Lorant, D. E., McIntyre, T. M. \& PrescotT, S. M. (1993). Juxtacrine intercellular signalling - another way to do it. Am. J. Resp. Cell Mol. Biol. 9, 573-577.

Ziv, T., Shimoni, Y. \& Mitrani, E. (1990). Activin can generate ectopic axial structures in the blastoderm explants. Development 115, 689-694.

\section{APPENDIX A}

\section{A1. Speed of the Wave in a Diffusive System}

We consider the equation

$$
v_{t}=s(x)+g(v)-\lambda v+D_{v} v_{x x},
$$

where $s(x)$ is a general localized source term, on the domain $[0, L]$ with zero-flux boundary conditions. To study travelling waves, we really need to consider an infinite domain. However, if we consider a sufficiently large domain with zero-flux boundary conditions, such that the boundaries do not affect the dynamics of the travelling wave, then we can simulate travelling wave profiles numerically. We assume that the effect of the source term is simply to trigger a travelling wave of inhibition and that the effects of the domain boundaries are small except in their immediate neighbourhood. We hence look for travelling wave solutions of eqn (A.1), without the source term, on an infinite domain.

Equation (A.1), without the source term, has homogeneous steady states which are the positive solutions of

$$
g(v)=\lambda v
$$

There are no biological data to guide us to the form that $g(v)$, the inhibitor production term, should take. Therefore, we consider the three typical functional forms that are commonly used as production terms in biology.

In the case represented by Fig. A1(a), 0 is the only homogeneous steady state and is stable. In this case, the inhibitor will just decay away from the source. For the form of $g(v)$ illustrated in Fig. A1(b), the zero steady state is unstable and any slight perturbation will cause convergence to the non-zero steady state. Travelling waves do occur in systems with this type of production term, but they will only do so if the level of inhibitor away from the source is exactly zero. If there is a noisy background of inhibitor concentration then this will grow to the non-zero homogeneous steady-state level. Hence, this case does not exhibit the necessary robustness required for the problem being studied here and we will ignore it in the subsequent analysis.

For the form of $g$ represented by Fig. A1(c), we look for travelling wave solutions of the form $v=v(z)$, where $z=x-c t, c$ is the speed of the wave and $v(z) \rightarrow 0$ as $z \rightarrow \infty, \quad v(z) \rightarrow v_{0}$ as $z \rightarrow-\infty . v_{0}$ is the stable non-zero homogeneous steady-state value. These boundary conditions imply that far ahead of the wave, the inhibitor concentration is zero, while far behind the front, the inhibitor concentration is at the steady state given by the largest intersection of the line $y=g(v)$ with the line $y=\lambda v$. In travelling wave coordinates, the equation is then

$$
D_{v} v_{z z}+c v_{z}+g(v)-\lambda v=0 .
$$
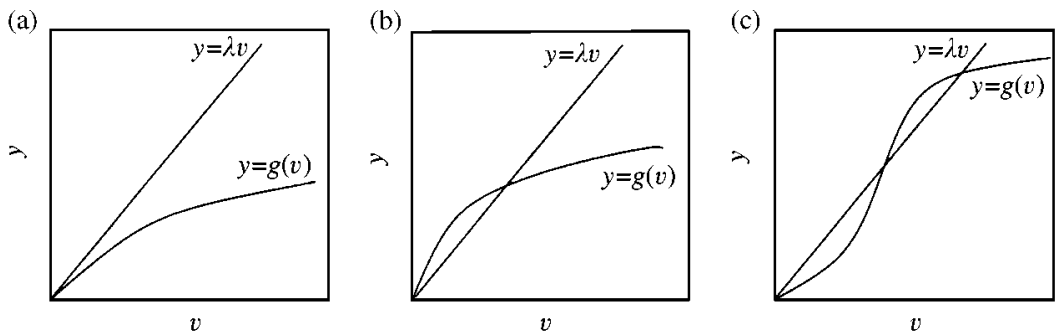

FIG. A1. Possible forms of $g(v)$, the self-activation for the inhibitor. $v$ is the concentration of inhibitor; $\lambda$ is its decay rate. 
For given $D_{v}, \lambda$ and $g$ of the form in Fig. A1(c), we can show, using standard phase-plane analysis, that there will be a solution for a unique travelling wave speed $c$.

For a given self-activation function, $g$, there is a maximal value of $\lambda$ given by

$$
\int_{0}^{v_{0}}(g(v)-\lambda v) \mathrm{d} v=0
$$

for which the inhibitory wave will propagate forward.

To obtain an estimate for the speed of propagation, we approximate $g(v)$ by a Heaviside function. This is equivalent to assuming that the uncommitted cells produce inhibitor at a fixed rate only when the background concentration exceeds a threshold. We discuss more realistic forms of $g$ later, although in some cases a sharp threshold response to cell signalling may be realistic, but this usually involves protein synthesis (Ferrell \& Machleder, 1998; Papin \& Smith, 2000).

With Heaviside function self-activation, eqn (A.3) becomes

$$
D_{v} v_{z z}+c v_{z}+\mu \mathscr{H}\left(v-v_{*}\right)-\lambda v=0,
$$

where

$$
\mathscr{H}\left(v-v_{*}\right)= \begin{cases}1, & v>v_{*}, \\ 0, & v<v_{*} .\end{cases}
$$

We assume that $v_{0}$ (the non-zero homogeneous steady-state value of $v) \equiv \mu / \lambda>v_{*}$. This means that there is a stable non-zero homogeneous steady state $v=v_{0}$. We assume that, in the travelling wave solution, $v \rightarrow 0$ as $z \rightarrow \infty$ and $v \rightarrow v_{0}$ as $z \rightarrow-\infty$.

We find that the wave speed is given by

$$
c=\sqrt{D_{v} \lambda}\left(\sqrt{\frac{v_{0}}{v_{*}}-1}-\frac{1}{\sqrt{\frac{v_{0}}{v_{*}}}-1}\right) .
$$

In particular, we can see that the wave will move forward iff $v_{0}>2 v_{*}$, which is equivalent to $\int_{0}^{v_{0}}\left[\mu \mathscr{H}\left(v-v_{*}\right)-\lambda v\right] \mathrm{d} v>0$. The travelling wave solution is given by

$$
v= \begin{cases}v_{*} \mathrm{e}^{\xi_{1}\left(z-z_{0}\right)}, & z>z_{0}, \\ \frac{\mu}{\lambda}+\left(v_{*}-\frac{\mu}{\lambda}\right) \mathrm{e}^{\xi_{2}\left(z-z_{0}\right)}, & z<z_{0},\end{cases}
$$

where $z_{0}$ is determined by the initial conditions and $\xi_{1}$ is the negative root and $\xi_{2}$ the positive root of

$$
D \xi^{2}+c \xi-\lambda=0
$$

Numerical simulations of the corresponding partial differential equation model for $v$ confirm this analytical prediction for the wave speed (Page, 1999).

\section{A1.1. HILL-FUNCTION SELF-ACTIVATION OF THE INHIBITOR}

If the inhibitor enhances its own production by binding to a receptor on the cell surface, then the rate of self-activated production of the inhibitor should reflect the level of receptor occupancy. We assume for now that the inhibitor production rate is simply proportional to the level of receptor binding. As the kinetics of receptor binding is likely to be rapid then the level of receptor binding will be approximated by $k_{1} v^{n} /\left(k_{2}+v^{n}\right)$, where $n$, the Hill coefficient, is the number of molecules of inhibitor that bind simultaneously to one receptor and $k_{1}$ and $k_{2}$ are constants that depend on the rate constants of the inhibitor-receptor binding (see Hill, 1910; Segel, 1991; Murray, 1989). Thus, we consider a self-activation term of this Hill function form. For $n$ large, the Hill function is similar to a Heaviside function. We now compare the ability to generate travelling waves and their speeds and forms in systems having a self-activation term which takes the form of a Hill function of order 2 or 3 with the corresponding properties of the system with Heaviside function dependence. If the self-activation terms takes the form of a Hill function of order 1 then the zero steady state of the inhibitor concentration is unstable, see Fig. A1(b), and any noise will trigger convergence to the non-zero steady state, so we assume this form is not relevant. A very large $n$ is unlikely from a physical point of view [although there have actually been found cases in which the apparent Hill coefficient, calculated 
from the dose-response curve, is as high as $n=35$, see Ferrell \& Machleder (1998), but this high apparent coefficient seems to rely on protein synthesis-dependent positive feedback, which would probably be slow] and anyway we expect extremely good agreement with the Heaviside solution, since the form of the Hill function tends towards a Heaviside function form as $n \rightarrow \infty$.

In order to compare like parameters with like in the Heaviside and Hill function equations we consider self-activation terms with the same inhibitor production rate at very high inhibitor concentration and the same inhibitor concentration at the point of maximum slope of $g$. The Hill function corresponding to $\mu H\left(v-v_{*}\right)$ is hence $\mu\left(v^{n} /\left(K v_{*}^{n}+v^{n}\right)\right)$, where $K=(n+1) /(n-1)$. This choice of matching is somewhat arbitrary, but gives functional forms which are fairly similar.

We simulate the Hill function equations, with $n=2$ and 3 , for the parameter values $v_{*}=1.0$, $\mu=10.0, \lambda=1.0$ and $D=0.0001$, chosen such that, according to our analysis for Heaviside function self-activation, a forward-propagating travelling wave solution exists and such that the diffusion coefficient is large enough to allow reasonably fast, accurate computation. We also match the analytical solution of the Heaviside function equation (dotted line) at $t=16$, to compare the forms of the solutions (see Fig. A2). For $n=2$, the solutions are similar except behind the wave, where the difference is largely due to different non-zero homogeneous steady-state values.
The agreement is better for $n=3$. The numerical simulations suggest a travelling wave speed of $c=0.019$ for $n=2$ and $c=0.023$ for $n=3$ compared to an analytical prediction (which agrees with the numerically calculated value) of $c=0.027$ for Heaviside function self-activation. Hence, the Hill function self-activation leads to a travelling wave of inhibition which is slower than that initiated with Heaviside function self-activation.

When we simulate the Hill function equation with $n=2$ or 3 and parameter values $v_{*}=2.0$, $\mu=5.0, \lambda=1.0$ and $D=0.001$ the inhibitor concentration collapses to zero. In both these cases, there is no non-zero homogeneous steady state. For a non-zero steady state to exist for $n=2$, we need $v_{*}<(1 / 2 \sqrt{3})(\mu / \lambda)$; however, this still does not ensure that the travelling wave solution propagates forwards. This will happen if $v_{*}<0.265 \mu / \lambda$ [using condition (A.4)], this being only slightly less than the value of $v_{*}$ required for a non-zero homogeneous steady state. For $n=3$, these values are more difficult to compute, but, as an approximation, we require $v_{*}<0.37 \mu / \lambda$ for a forward propagating wave. These values compare with the condition $v_{*}<\frac{1}{2} \mu / \lambda$ for the Heaviside function equation. Thus, the more receptor binding sites involved in the inhibitor-receptor binding, i.e. the higher the value of $n$ and the more like a Heaviside function the self-activation, the more robustly the system initiates a forward propagating wave of inhibition.
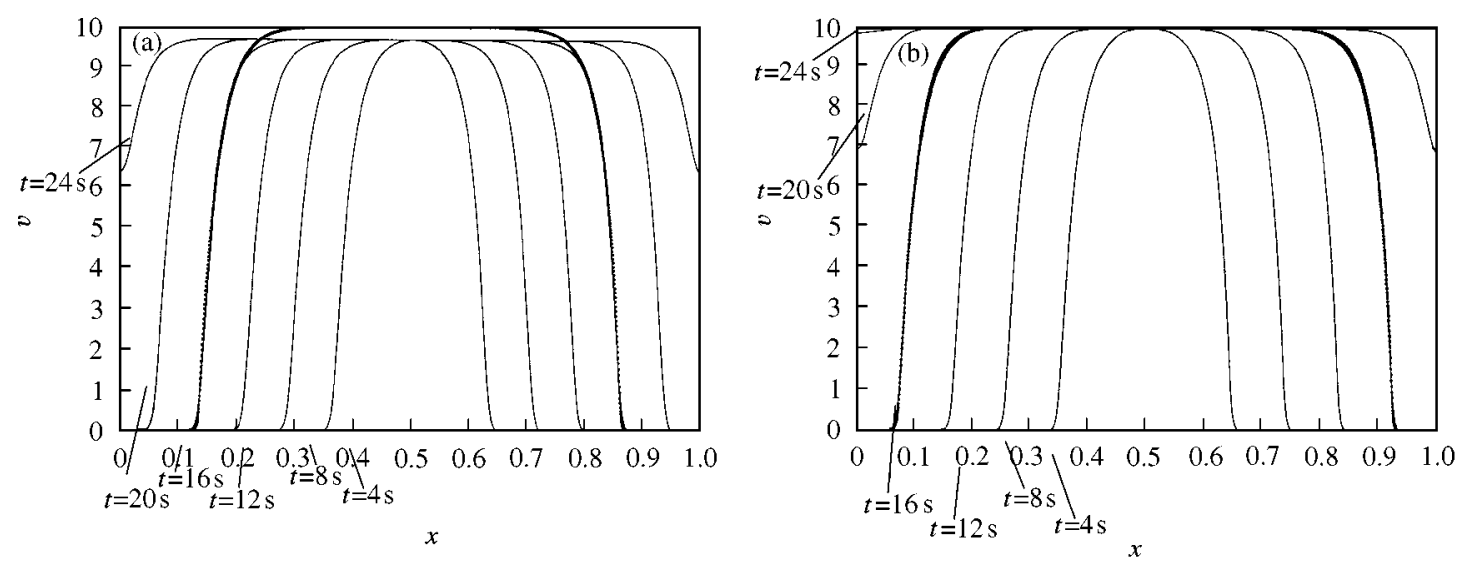

FIG. A2. Numerical solutions of the inhibitor equation with Hill function self-activation $(-)$ and analytical form of the solution with Heaviside self-activation $(\cdots)$ - see text for details. Parameters as in Fig. 3 - exact form of Hill function given in text. Output times shown in the figure. (a) Hill function of order 2. (b) Hill function of order 3. In both cases, a spatial discretization of 2001 points was used. 


\section{A1.2. REALISTIC PARAMETER VALUES}

In our primitive streak initiation model, we want only one streak to be initiated in any connected piece of blastodisc. This will happen if the travelling wave of inhibition is sufficiently fast. From both our simulations and our analytical form of the wave speed in the Heaviside function inhibitor equation, we see that the inhibitory wave speed increases with the ratio $v_{0} / v_{*}$, where $v_{0}=\mu / \lambda$. We thus consider how large this ratio could plausibly be. As the ratio gets large, a non-zero homogeneous steady state will always exist and will take a value approximately equal to $v_{0}$. Thus, $v_{0} / v_{*}$ corresponds approximately to the ratio of the saturation concentration of inhibitor to the threshold value at which the non-differentiated cells begin to produce inhibitor. It has been shown in certain morphogenetic systems that cells respond to threshold levels of chemicals which are very far from the saturation levels [see Dyson \& Gurdon (1998), although it should be noted that this paper refers to low receptor occupancy and not to a saturation level given in terms of kinetic parameters]. Here we shall take a generous estimate of the maximum likely value of this ratio as being 100; less generous estimates would predict slower waves of inhibition. We assume that the half-life of the inhibitor in vivo will be at least $10 \mathrm{~s}$, leading to a maximum value of $\lambda$ of $0.1 \mathrm{~s}^{-1}$. Since we are not interested in the absolute values of the inhibitor concentration, we take $v_{*}$ to be 1 in some arbitrary units. This means that $\mu \leqslant 10.0$ concentration units per second. We assume that the inhibitor is a protein of average mass and that its diffusion coefficient will be $\leqslant 1.0 \times 10^{-7} \mathrm{~cm}^{2} \mathrm{~s}^{-1}$.

If we allow all the parameters to take their bounding values then we obtain an analytical wavespeed for the system with Heaviside self-activation of approximately $10^{-2} \mathrm{~mm} \mathrm{~s}^{-1}$. Thus, the inhibitory wave would pass from the posterior of the blastoderm to the anterior in less than about $5 \mathrm{~min}$.

Since the ratio $v_{0} / v_{*}$ is large we expect the behaviour of the system with Hill function self-activation to be similar to that with Heaviside self-activation, although the wave will be a little slower. We simulate the system numerically with Heaviside function and Hill function of order 2 self-activation on a periodic domain of

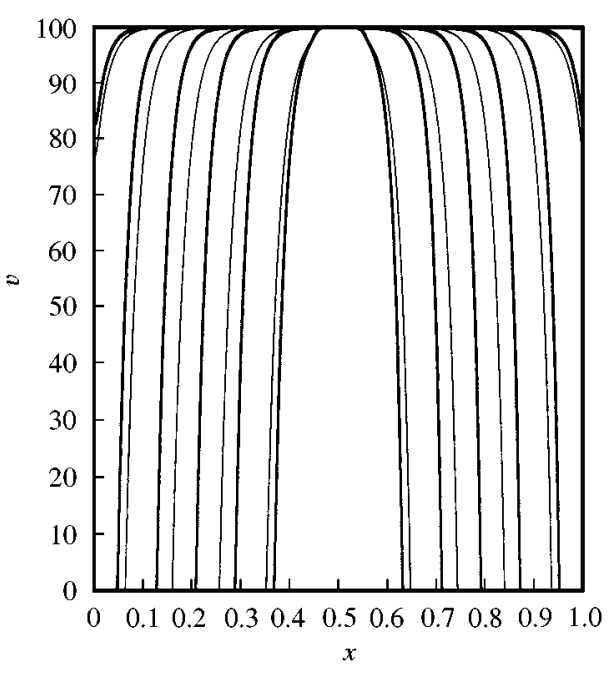

FIG. A3. Numerical solutions of the inhibitor equation with Hill function self-activation $(-)$ and Heaviside self-activation ( - ) at times $t=40,80,120,160,200$ and $240 \mathrm{~s}$. Realistic parameter values of $D=1.0 \times 10^{-7} \mathrm{~cm}^{2} \mathrm{~s}^{-1}$, $\mu=10.0$ concentration units $\mathrm{s}^{-1}, \lambda=0.1 \mathrm{~s}^{-1}$ and $v_{*}=1.0$ concentration unit are taken on a periodic domain of length $4 \mathrm{~mm}$. A spatial discretization of 8001 points was used.

length $4 \mathrm{~mm}$, with the parameters taking their bounding values (see Fig. A3). We see that in the Heaviside function case, the wave takes less than $200 \mathrm{~s}$ to reach the anterior of the domain (we consider the middle of our simulation to correspond to the posterior and the edges to correspond to the anterior) and in the Hill function case less than $240 \mathrm{~s}$. This shows very good agreement with the analytical estimate.

\section{A1.3. INHIBITORY TRAVELLING WAVES IN TWO DIMENSIONS}

Experiments involving manipulation of the blastodisc suggest that the wave of inhibition should pass through the central disc rather than be confined to the marginal zone. We are interested therefore in whether the propagation of a wave in two dimensions differs significantly in speed from that in one dimension. The eikonal equation (Grindrod, 1996) shows that in the case of radially symmetric propagation, the wave propagates at rate which is an amount $D / r$ slower than the one-dimensional speed, where $D$ is the diffusion coefficient of the inhibitor and $r$ is the radial coordinate. Biologically, realistic diffusion coefficients are very small and so at a very short 
distance from the source of the wave the difference between the one- and two-dimensional speeds should become negligible. Thus, if we assume that the source is not perfectly localized (and so outside the source $r \gg 1 / D$ ), then the time taken for the inhibitory wave to propagate in one dimension should give us reasonable estimates for the two-dimensional case. Numerical simulations on a two-dimensional domain confirm this result (Page, 1999).

We conclude, therefore, that a cell-inhibitory model in which the inhibitor diffuses in the classic Fickian sense can exhibit wave speeds sufficiently fast to account for the inhibition of streak initiation observed experimentally.

\section{A2. Wave Speed in a Juxtacrine Relay System}

\section{A2.1. ANALYSIS OF THE CASE WITH HEAVISIDE FUNCTION SELF-ACTIVATION}

We assume that the level of signalling depends on the average of the inhibitor concentration in the cell's immediate neighbours and that the inhibitor decays linearly. The model equations are as follows:

$$
\begin{aligned}
&\left(v_{i}\right)_{t}=-\lambda v_{i} \\
&+\mu \mathscr{H}\left[\frac{1}{2} v_{i-1}(t-\sigma)+\frac{1}{2} v_{i+1}(t-\sigma)-v_{*}\right], \\
& v_{i}(t)=\frac{\mu}{\lambda} \quad \text { as } i \rightarrow-\infty \text { for any fixed } t, \\
& v_{i}(t) \rightarrow 0 \quad \text { as } i \rightarrow \infty \text { for any fixed } t,
\end{aligned}
$$

where $\mathscr{H}$ is the Heaviside function, $v_{i}$ is the concentration of inhibitor in cell $i, v_{*}$ is the threshold concentration which causes a cell to start producing inhibitor and $\sigma$ is the delay between signalling to a cell and the upregulation of the signal presented by that cell. The first equation has uniform steady-state solutions, $v_{i}(t)=0$ and $v_{i}(t)=\mu / \lambda, \forall i$ and $t$. For a travelling wave in a cellular system, we want the concentration in cell $i$ at time $t$ to be the same as that in cell $i-1$ at a certain fixed delay before, i.e. $v_{i}(t)=v_{i-1}(t-\tau), \quad \forall i$ and $t$. This gives a wavespeed of $s / \tau$, where $s$ is the cell separation.
Substituting this into eqn (A.9) gives

$$
\begin{aligned}
\left(v_{i}\right)_{t}= & -\lambda v_{i}+\mu \mathscr{H}\left[\frac{1}{2} v_{i}(t+\tau-\sigma)\right. \\
& \left.+\frac{1}{2} v_{i}(t-\tau-\sigma)-v_{*}\right] .
\end{aligned}
$$

We assume that initially $v_{i}=0$ for $i>0$ and so $v_{i}$ will always be zero in front of the wave (i.e. before $v_{i-1}$ reaches threshold), for $i>0$. Thus, in this simple scenario, only the signal from the left-hand side matters.

Now when $\left(\frac{1}{2}\right) v_{i}(t+\tau-\sigma)>v_{*},\left(v_{i}\right)_{t}=-\lambda v_{i}$ $+\mu$, so

$$
v_{i}=\frac{\mu}{\lambda}-B \mathrm{e}^{-\lambda t}
$$

This must match with the zero solution when $\frac{1}{2} v_{i}(t+\tau-\sigma)=v_{*}$. Let the time when the match occurs be denoted by $t_{*}(i)$, then

$$
\begin{gathered}
v_{i}(t)= \begin{cases}\frac{\mu}{\lambda}\left(1-\mathrm{e}^{-\lambda\left(t-t_{*}(i)\right)}\right), & t>t_{*}(i), \\
0, & t<t *(i),\end{cases} \\
\frac{1}{2} v_{i}\left(t_{*}(i)+\tau-\sigma\right)=v_{*} .
\end{gathered}
$$

The first and third of these give us

$$
\tau=\sigma+\frac{1}{\lambda} \ln \left(\frac{1}{1-\left(2 v_{*} / v_{0}\right)}\right),
$$

where $v_{0}=\mu / \lambda$. The speed is then $s / \tau$. This is in good agreement with numerical simulations of eqn (A.9). (Note, for $\lambda \ll \mu / v_{*}$, the speed is almost independent of $\lambda$, with $\tau \approx \sigma+2 v_{*} / \mu$.)

As with the diffusive travelling wave, more realistic Hill function self-activation of the inhibitor leads in general to a slower wave. For some parameter values, a travelling wave may be established in the case of Heaviside function self-activation, but not for Hill function selfactivation (Page, 1999).

\section{A2.2. THE EFFECT OF $\sigma$}

Analysis of the juxtacrine relay inhibitor equation with Heaviside self-activation indicates that for fixed $\lambda, \mu$ and $v_{*}$ there is a maximal speed 
which occurs for $\sigma=0$. The time taken, $\tau$, for the wave to pass from one cell to the next is given by

$$
\tau(\sigma)=\tau(0)+\sigma
$$

Thus, a delay between inhibitor signalling to the cells and the production of more inhibitor translates simply into a delay in the wave of inhibition passing from one cell to the next. $\mathrm{Nu}-$ merical simulations indicate that the same relation between $\tau$ and $\sigma$ holds (approximately) when the self-activation takes the Hill function form (data not shown).

\section{A2.3. INHIBITORY TRAVELLING WAVES IN TWO DIMENSIONS}

The dynamics of juxtacrine signalling systems in two dimensions are dependent on the positions of the cells and the number and orientation of nearest-neighbour interactions. In the following simulations, we assume that cells are arranged in a square lattice and that they signal only to those neighbours with which they share a side. We also assume that a cell presents one-quarter of its quota of inhibitor on each side. Thus, the level of inhibitor presented to the cell at position $(i, j)$ is given by

$$
\frac{1}{4}(v(i+1, j)+v(i-1, j)+v(i, j+1)+v(i, j-1)) .
$$

For ease of simulation, we assume that $\sigma=0$. Our previous results suggest that a non-zero $\sigma$ simply adds a delay to the time taken for the wave to move from one cell to the next.

Figure A4(a) shows the position of the wavefront at $t=5, t=10, \ldots, t=45$ (by $t=50$ it has covered the entire domain) in a numerical simulation of the relay inhibitor equation on a square grid of $101 \times 101$ cells with Heaviside self-activation and parameter values $\mu=10.0, \lambda=1.0$ and $v_{*}=1.0$. The cells are indexed by pairs $(i, j)$ denoting their positions in the two directions parallel to the edges of the square. The inhibitor concentration initially has value $\mu / \lambda$ for cells with $j \leqslant 10$ and $46 \leqslant i \leqslant 56$. The geometry of the lattice means that the wavefront approximately preserves its initial square form. The speed of the travelling wave is less than half of that in the one-dimensional case. This reduction in speed is largely due to the fact that the cells now share their signalling between four neighbours rather than two and this reduces the amount of signalling received by a cell at the front from its neighbour behind the front.

Figure A4(b) shows the position of the wavefront at $t=5, t=10, \ldots, t=50$ in a simulation of the relay inhibitor equation with Hill function order 2 self-activation. The other details of the simulation are as for Fig. A4(a). In this case, the wavefront becomes much more rounded (this seems to be due to the fact that it is not sharp). The travelling wave is again much slower than in one dimension.
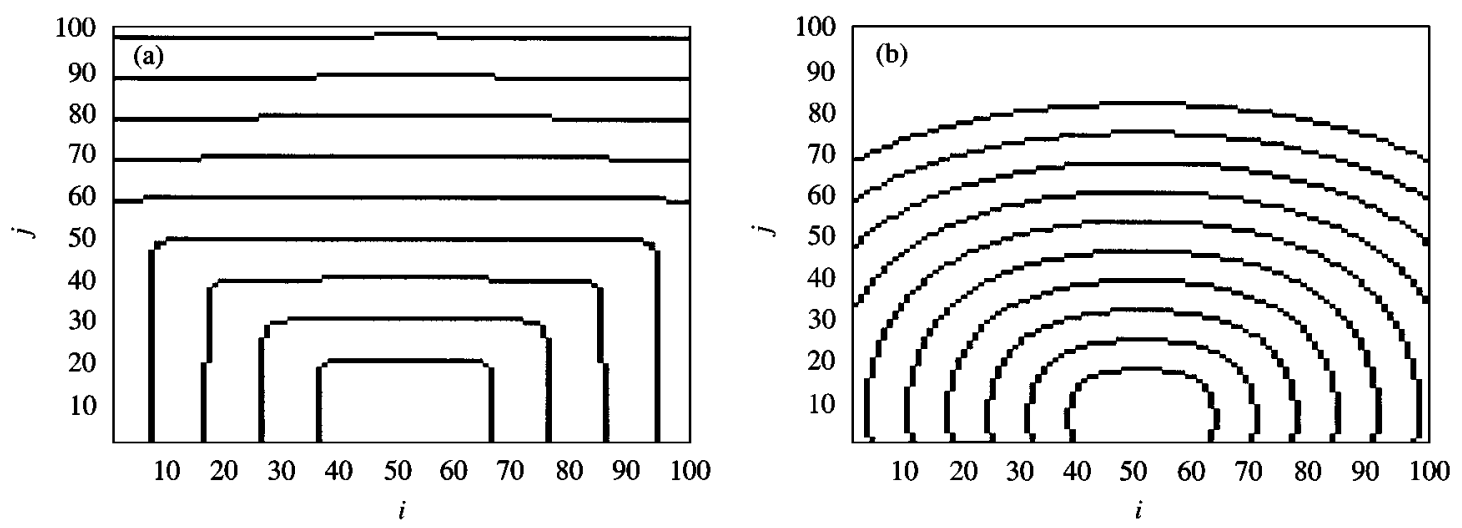

FIG. A4. Position of wavefronts at 5 time unit intervals in numerical simulation of the relay inhibitor equation on a domain of $101 \times 101$ cells. (a) Heaviside function self-activation, $\mu=10.0, \lambda=1.0$ and $v_{*}=1.0$ as in Fig. 3. For details, see text. (b) As (a) but with Hill function order 2 self-activation. 


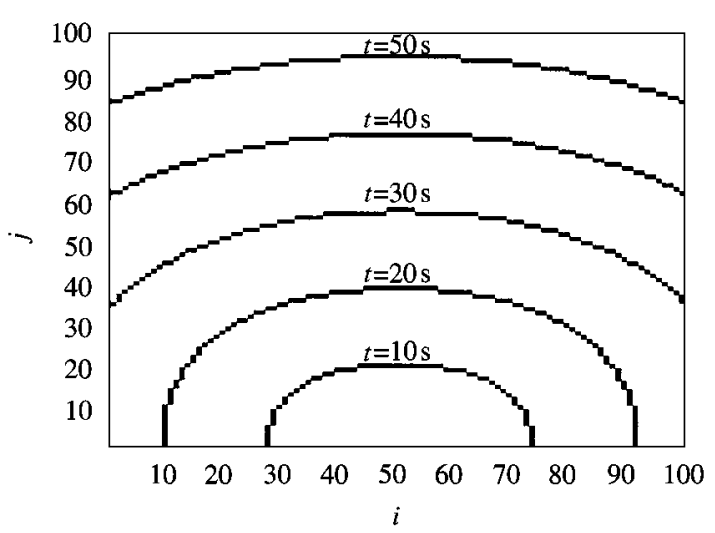

FIG. A5. Position of wavefronts at $10 \mathrm{~s}$ intervals in a numerical simulation of the relay inhibitor equation with Hill function order 2 self-activation on a domain of $101 \times 101$ cells. "Realistic" parameter values $-\lambda=0.1 \mathrm{~s}^{-1}$, $\mu=10.0 v_{*} \mathrm{~s}^{-1}$. For details see text.

\section{A2.4. REALISTIC PARAMETERS}

As in the case of the diffusive wave, we try to assess how fast a wave of inhibition might propagate in the juxtacrine signalling system. As before, we take parameter values $\lambda=0.1 \mathrm{~s}^{-1}$, $v_{*}=1$ in some concentration units and $\mu=10.0 v_{*} s^{-1}$. We simulate the system with Hill function order 2 self-activation on a square domain. The blastodisc has a diameter of about $2 \mathrm{~mm}$ which corresponds to roughly 500 cell lengths. We simulate on a smaller domain of $101 \times 101$ cells, in order to limit the time taken to perform the simulations. For ease of simulation we assume that $\sigma=0$ and allow for an additional time of approximately $100 \sigma$, where $\sigma$ is the actual delay, for the wave to propagate. The position of the wavefront at $10 \mathrm{~s}$ intervals is shown in Fig. A5. Initially, it is assumed that only cells adjacent to the posterior marginal zone are activated and so we set $v(i, j)=\mu / \lambda$ for $j=1$ and $45 \leqslant i \leqslant 55$ and $v(i, j)=0$ elsewhere. The wave takes less than $60 \mathrm{~s}$ to propagate throughout the domain. We can assume, since the wave propagates at approximately constant speed, that it will take less than $5 \mathrm{~min}$ to propagate across a domain of $500 \times 500$ cells.

The magnitude of the delay $\sigma$ depends on the mechanism by which cells upregulate their surface inhibitor concentration in response to receptor signalling (see Monk, 1998). A mechanism involving gene transcription could cause a delay of as much as several minutes (too slow for the purposes of this model). Release of inhibitor from intracellular vesicles is likely to take the order of a minute (or a little less). This again would probably be too slow and certainly these delays would dominate the time taken for the wave to propagate. A faster mechanism would be the conversion of inhibitor from an inactive to an active form via protein cleavage (see Lyons et al., 1988). This could take less than a second and hence the inhibitory wave could propagate across the domain in around $10 \mathrm{~min}$.

These calculations suggest that a juxtacrine signalling mechanism is also able to propagate fast inhibitory waves. 